\title{
Analysis and Optimized Design of a Distributed Multi-Stage EMC Filter for an Interleaved Three-Phase PWM-Rectifier System for Aircraft Applications
}

\author{
Nico Hensgens, Marcelo Silva, Jesús A. Oliver, Pedro Alou, Oscar Garcia, José A. Cobos
}

\begin{abstract}
Different possible input filter configurations for a modular three-phase PWM rectifier system consisting of three interleaved converter cells are studied. The system is designed for an aircraft application where MIL-STD-461E conducted EMI standards have to be met and system weight is a critical design issue. The importance of a LISN model on the simulated noise levels and the effect of interleaving and power unbalance between the different converter modules is discussed. The effect of the number of filter stages and the degree of distribution of the filter stages among the individual converter modules on the weight and losses of the input filter is studied and optimal filter structures are proposed.
\end{abstract}

Index Terms-EMC, EMI, input filter, optimization, threephase, PWM rectifier, MIL-STD-461E, LISN

\section{INTRODUCTION}

Power electronic converters are sources of electromagnetic interferences (EMI) where it originates from semiconductor switches operating at high frequency [1], [2], [3]. In order to prevent disturbances in other appliances connected to the same grid, international standards have been established which define the maximal levels of EMI that power converters are allowed to emit. Depending on the application area, different standards are applicable. In case of industrial or residential environments, the Class A and Class B limits as defined in the CISPR standards [4], [5] have to be met. In case of aerospace applications, the military standard MIL-STD-461E [6] is applicable.

Besides the use of other EMI mitigation techniques [7], in general an EMI input filter is necessary to comply with the standards. The filter however contributes significantly to the weight and volume of the power converter system [8] as well as to its losses. Therefore an optimized design is desirable, especially in aerospace applications, where a low weight of the converter system is of high importance.

This paper presents the analysis and optimized design of the EMC input filter for a three-phase converter system for aerospace applications. The converter consists of three individual and identical modules. This allows for an additional degree of freedom in the input filter design space, i.e. the degree of

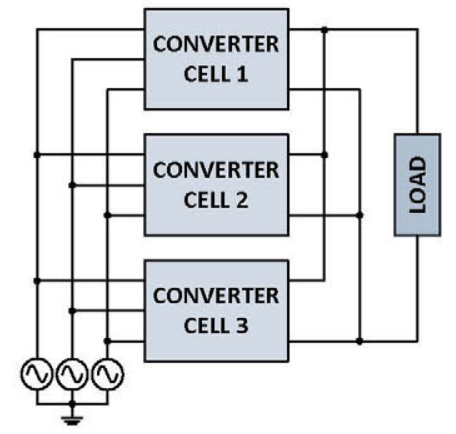

Fig. 1. Simplified schematic of the converter system consisting of three modular converter cells

distribution of the input filter between the individual converter modules. The effect of a distributed design of the input filter and the number of filter stages on the expected weight and losses is analyzed and an optimized design is proposed. Additionally an issue regarding the influence of the EMC measurement equipment, i.e. the line impedance stabilization network (LISN) is addressed, which plays an important role when performing converter simulations to obtain the converter input current spectrum.

\section{Converter System}

A modular three-phase power converter system has been designed for an aircraft application. The system consists of three individual converter cells which are connected in parallel and operated in an interleaved mode, i.e. with a phase shift of 120 degrees with respect to each other. Each individual cell consists of a three-phase buck-type PWM rectifier [9], together with a voltage-fed phase-shifted full-bridge to regulate the output voltage and provide galvanic isolation. The basic converter structure is shown in Fig. 1 and Table I summarizes the main converter specifications. Fig. 2 shows a more detailed schematic of one single converter cell. 


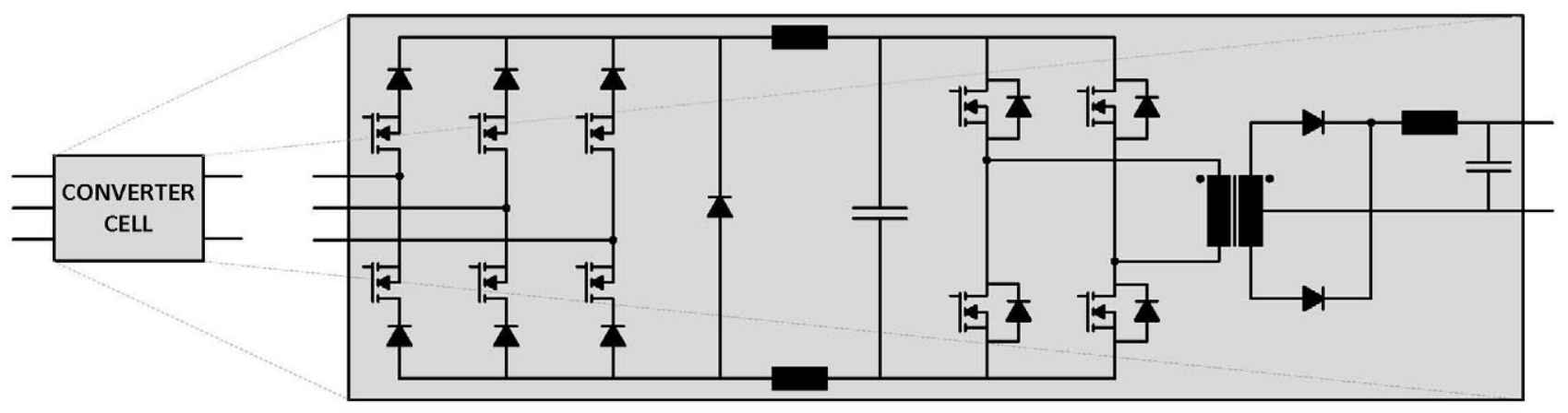

Fig. 2. Detailed view of an individual converter cell consisting of a three-phase buck-type PWM rectifier and a voltage-fed full-bridge converter

TABLE I

CONVERTER SPECIFICATIONS

\begin{tabular}{|l|c|}
\hline Input Voltage $V_{i n}$ & $115 V_{r m s} / 400 \mathrm{~Hz}$ \\
\hline Output Voltage $V_{o u t}$ & $270 V_{d c}$ \\
\hline Nominal Output Power $P_{o}$ & $10 \mathrm{~kW}(3 \times 3.3 \mathrm{~kW})$ \\
\hline Rectifier Switching Frequency $f_{s w, R e c t}$ & $60 \mathrm{kHz}$ \\
\hline Full-bridge Switching Frequency $f_{s w, F B}$ & $180 \mathrm{kHz}$ \\
\hline
\end{tabular}

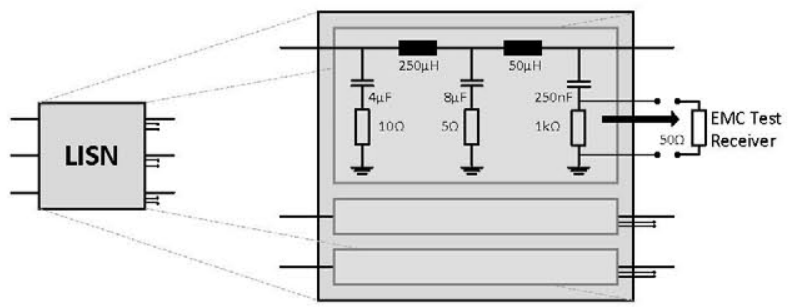

Fig. 3. Three-phase LISN model (only one of the three phases is shown in detail)

\section{EMC SIMULATIONS AND MIL-STD-461E}

The conducted emission levels of the converter system have been obtained through simulation, in order to start the input filter design before a working prototype was available. In all converter simulations a model of the line impedance stabilization network (LISN) as defined in [6] and shown in Fig. 3 has been included, connected between the threephase source and the power converter. Two different input noise spectra have been calculated, one time via an FFT of the current as measured at the input port of the converter system multiplied by a $50 \Omega$ standard resistance, and one time as a voltage drop over the same standard resistance inside the LISN. The two methods are depicted schematically in Fig. 4. In Figs. 5a and 5b the input current spectrum of the converter system is shown as obtained by method one and method two respectively. One can clearly see that in the frequency range between $10 \mathrm{kHz}$ and several $100 \mathrm{kHz}$ the noise levels measured by the LISN are lower than those considering the converter input current directly. This fact is due to the impedance characteristic of the LISN which is

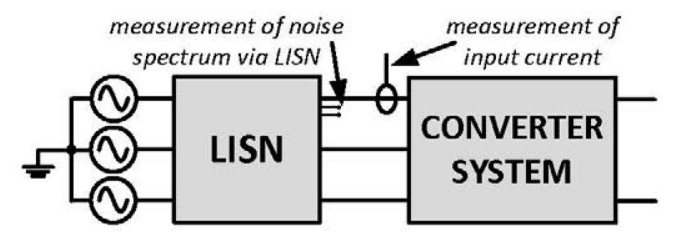

Fig. 4. Converter system with LISN and two methods of obtaining the input current spectrum

shown in Fig. 6. At the rectifier switching frequency of 60 $\mathrm{kHz}$, the LISN impedance is approximately $17 \Omega$ instead of $50 \Omega$. Thus the noise levels measured in the LISN at $60 \mathrm{kHz}$ are $20 \log \left(\frac{17}{50}\right) \simeq-9 d B \mu V$ lower than those obtained from the direct measurement of the input current. A design based on the current spectrum as measured at the converter input port would lead to an oversized and thus suboptimal input filter. This is often neglected in applications where only the frequency range $150 \mathrm{kHz}-30 \mathrm{MHz}$ is considered. In that case the effect can effectively be neglected since its influence is very small. In the frequency range $10 \mathrm{kHz}-10 \mathrm{MHz}$, i.e. for aircraft applications, it needs however to be considered.

\section{EMC InPUT FILTER DESIGN}

Conducted EMI standards for industrial or residential equipment cover the frequency range of $150 \mathrm{kHz}-30 \mathrm{MHz}$ [5] which allows ignoring all switching frequency harmonics below $150 \mathrm{kHz}$ in the design of the input filter [10]. This converter system however has to comply with military conducted EMC standards [6] which cover the frequency range of 10 $\mathrm{kHz}-10 \mathrm{MHz}$. Unless a very low switching frequency below $10 \mathrm{kHz}$ is selected, the first input current harmonic needs to be considered for the input filter design. Moreover the line frequency in aerospace applications is $400 \mathrm{~Hz}$ which in case of a low cutoff frequency of the designed filter may lead to a deterioration of the system power factor [12] and renders unfeasible the use of too low cutoff frequencies leading to a high total harmonic distortion. This puts significant constraints 

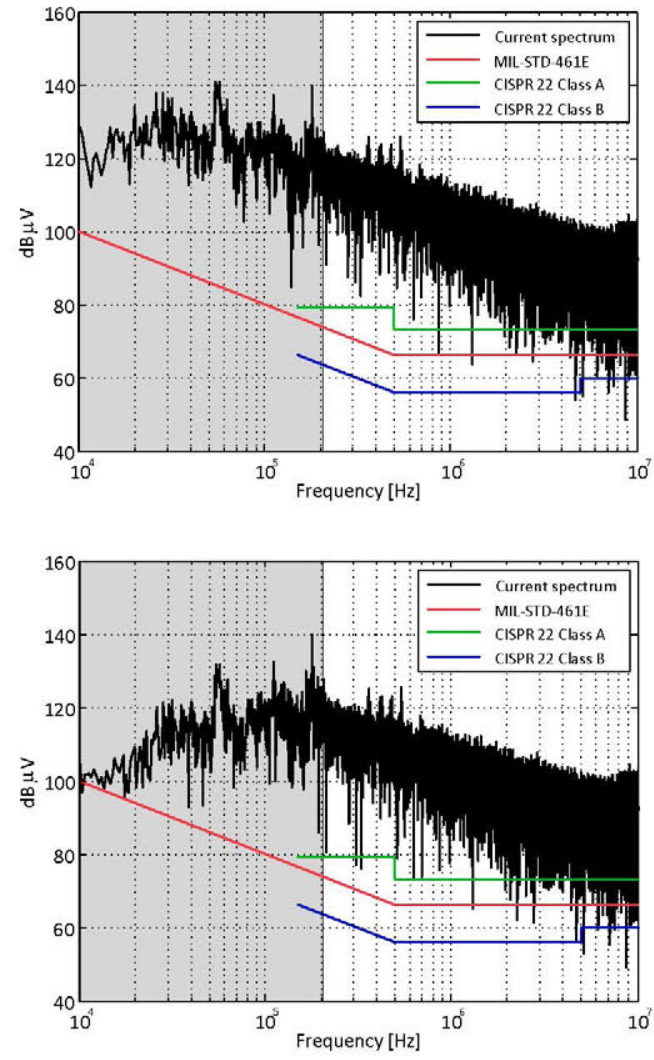

Fig. 5. Simulated DM noise spectrum of the converter system: obtained by measuring the converter input current (top) and via the LISN (bottom).

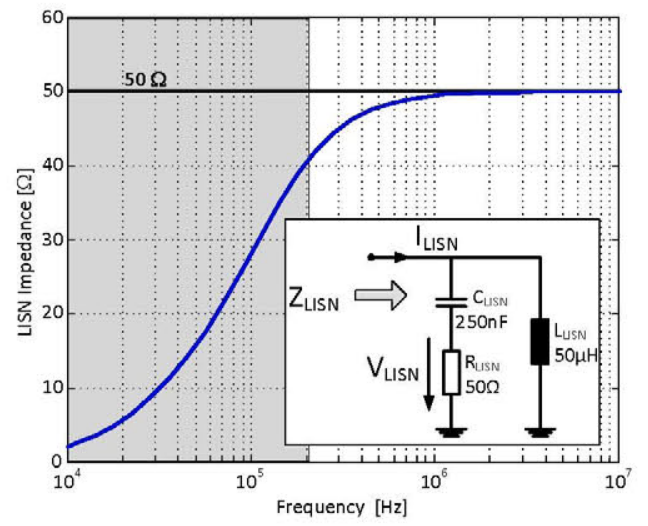

Fig. 6. Impedance characteristic of the LISN (a simplified LISN model as shown in the Figure has been used)

on the frequency range in which the filter cutoff frequency can be placed, as depicted in Fig. 7.

\section{A. Filter Distribution}

In the following we will analyze the influence of the number of input filter stages and and their degree of distribution on the total filter weight. Note that every differential mode (DM) filter stage consists in its basic configuration, i.e. without additional

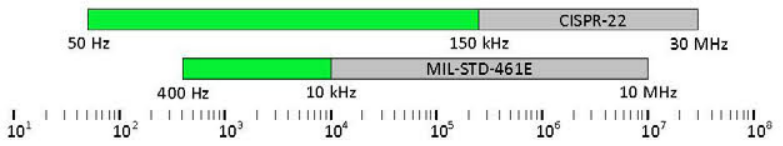

Fig. 7. Frequency range between grid frequency and frequency range considered by conducted EMI standards for residential/industrial and aerospace applications respectively.

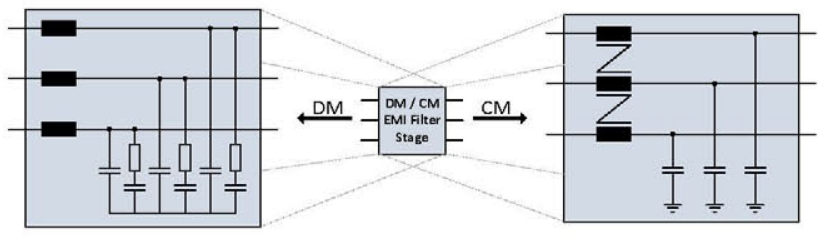

Fig. 8. Differential mode (DM) and common mode (CM) filter stage

damping elements, of three DM chokes and three line-to-line capacitors (X-capacitors) and every common mode (CM) filter stage consists of one three-phase $\mathrm{CM}$ choke and three lineto-ground capacitors (Y-capacitors). Additional RC damping networks have been included in every DM filter stage to provide damping of the filter resonance [13][14]. This is shown in Fig. 8. Also other damping networks than the parallelRC network could be considered. However other topologies, e.g. series-RL or parallel-RL damping [13], add an additional three inductors per filter stage, whereas the RC network adds capacitors with a considerably lower weight. Since it is our goal to design a filter with minimal weight, the RC damping presents the best solution.

The input filter for this converter system can be distributed in different ways. Either one common input filter can be designed for all three converter cells or individual input filters for every single converter cell. In case of a multi-stage filter, also a distributed filter structure with individual filters as well as a common filter can be considered. These three different filter structures are depicted in Fig. 9. The notation " $\mathrm{N}+\mathrm{M}$ " is proposed to describe the degree of distribution of the input filter. $\mathrm{N}$ denotes the number of filter stages which are common for all converter cells and $M$ the number of filter stages for every individual converter cell. For example " $1+2$ " denotes a three stage filter with one common filter stage for all three cells and two individual filter stages in front of every converter cell.

\section{B. DM Filter Design}

In case of a perfectly balanced system and considering the fact that the three converter cells work in interleaved operation with a phase shift of 120 degrees with respect to each other the first two switching frequency harmonics at $f_{s w, \text { Rect }}=60 \mathrm{kHz}$ and $2 f_{\text {sw, Rect }}=120 \mathrm{kHz}$ are canceled out. In the converter specifications however a maximal power unbalance of up to $10 \%$ is specified, i.e. one converter cell provides $110 \%$ of its nominal power $P_{o} / 3$ and the other two a power of $95 \%$ of 


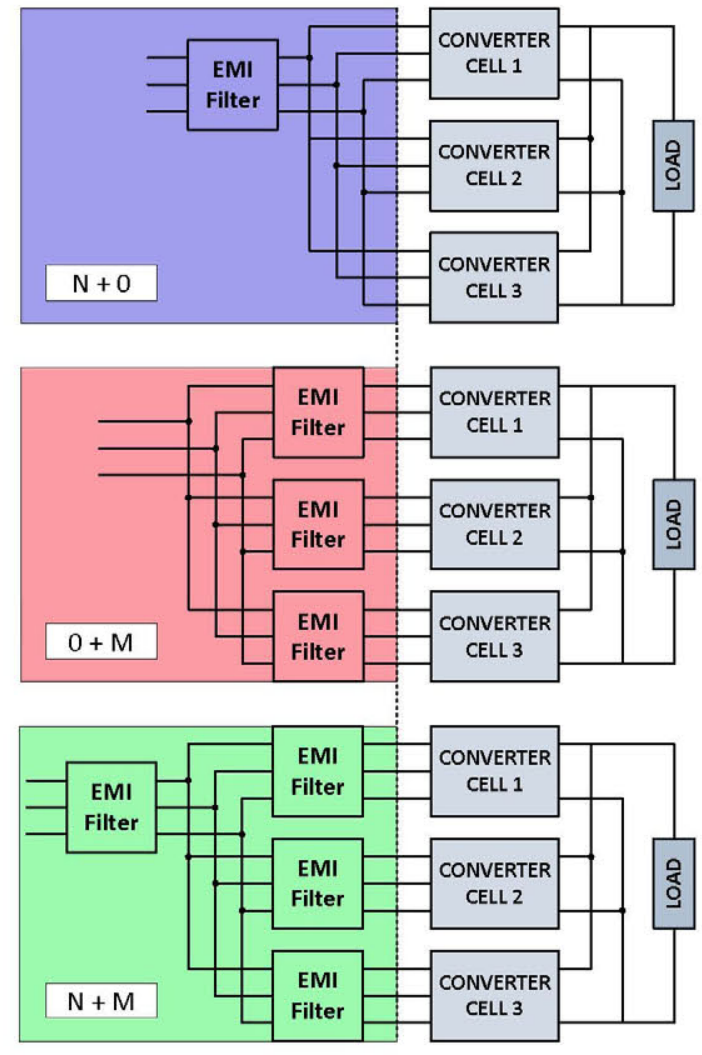

Fig. 9. Different degrees of distribution of the input filter: totally concentrated filter " $\mathrm{N}+0$ " (blue), totally separated filter " $0+\mathrm{M}$ " (red) and distributed filter "N+M" (green)

their nominal power $P_{o} / 3$. For the design of the input filter this worst case operating point needs to be considered. In that case these first switching frequency harmonics are not canceled out anymore and a significant noise peak appears at the frequency $f_{s w, R e c t}=60 \mathrm{kHz}$. The noise spectra of the converter system without interleaving, the system with interleaving and perfect power balance and the system with interleaving and a 10\% power unbalance are shown in Fig. 10 respectively. It can be seen that in the system without interleaving, noise peaks appear at all switching frequency harmonics. In the interleaved system, the amplitude of the first two noise peaks is considerably reduced and the most important noise peak appears at the third switching frequency harmonic at $3 f_{s w, R e c t}=180 \mathrm{kHz}$. In the unbalanced system a critical noise peak reappears at $f_{s w, R e c t}=60 \mathrm{kHz}$ with an amplitude of $143 \mathrm{~dB} \mu \mathrm{V}$ (compared to $149 \mathrm{dB \mu V}$ in the completely unbalanced system).

The design of the DM filter is based on the spectrum shown in the bottom picture of Fig. 10. The cutoff frequencies of the filters have been calculated such as to distribute the required attenuation equally over all filter stages. This design where all filter stages provide the same amount of attenuation leads to a simple design where all filter stages consist of the same components (L'a and Cs) and also to a design with
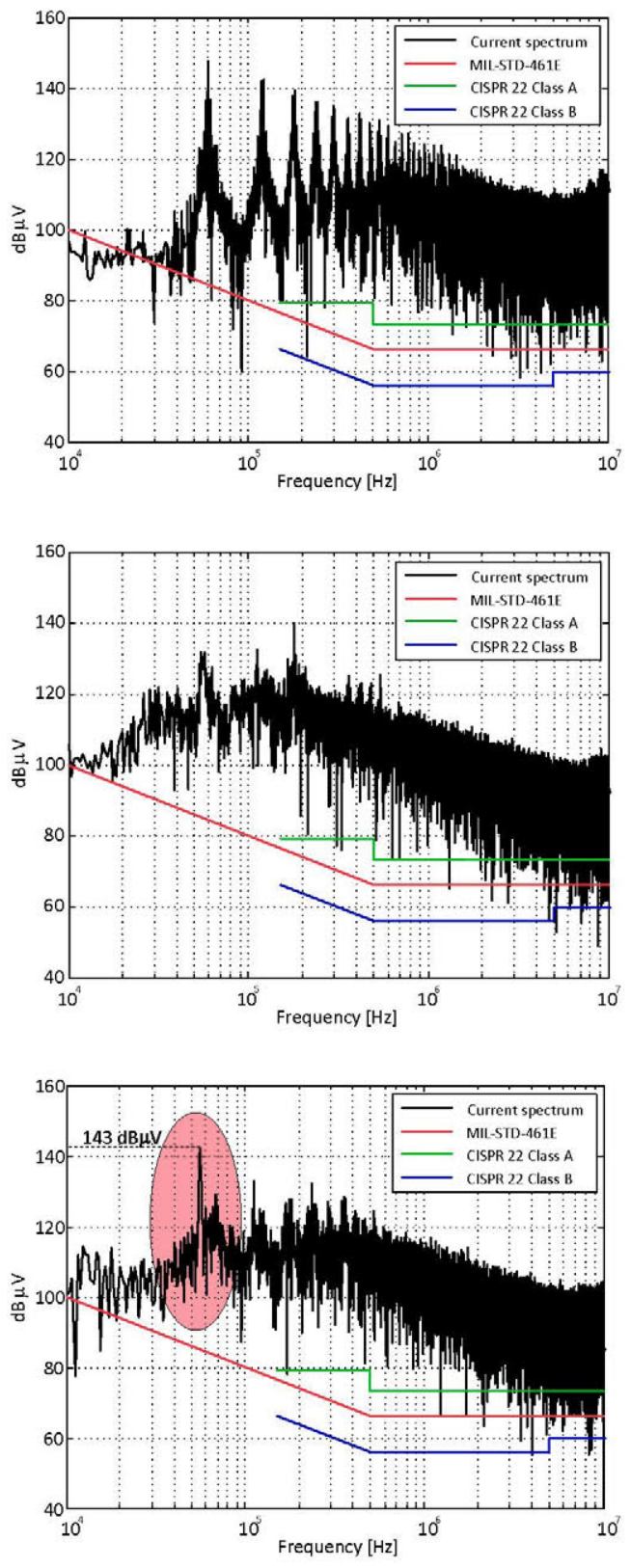

Fig. 10. Input current spectra of the converter in case of a system, without employing interleaving (top), an interleaved and perfectly balanced system (middle) and an interleaved system with a power unbalance of $10 \%$ (bottom).

minimal volume and weight [11]. The individual filter stages are designed as follows:

First the cutoff frequency $\omega_{\text {cutoff }}$ of the individual filter stages are calculated according to

$$
\omega_{\text {cutoff }}=\frac{2 \pi 60 k H z}{\sqrt{10^{a t t / 20}}}=\frac{1}{\sqrt{L C}}
$$

where att is the attenuation (in $d B \mu V$ ) the filter stage under consideration has to provide and $\mathrm{L}$ and $\mathrm{C}$ are the inductance 
and capacitance values used in that same filter stage. In order to obtain the values of $\mathrm{L}$ and $\mathrm{C}$ first a capacitance value is calculated in order to minimize the effect of the filter stage on the converter power factor [12]:

$$
C=\frac{1}{R_{L} \sqrt{\omega_{\text {cutoff }}^{2}-\omega_{\text {grid }}^{2}}}
$$

where $\omega_{\text {grid }}$ is the grid frequency, i.e. $400 \mathrm{~Hz}$ for aircraft applications and $R_{L}$ is the load impedance of the input filter (in case of a PFC converter a simple resistive model can be used). Then a capacitor $C_{d}$ with the closest commercially available value is selected and finally in order to achieve the required attenuation, the inductance is calculated as

$$
L=\frac{1}{\omega_{\text {cutoff }}^{2} C_{d}}
$$

\section{CM Filter Design}

The filter design has been performed without access to the final converter layout, which made an estimation of the parasitic capacitance to ground and thus an estimation of the prospected $\mathrm{CM}$ noise levels very difficult. It has been decided to include two CM filter stages in the input filter to provide sufficient attenuation for CM noise. Additionally only commercially available three-phase nanocrystalline CM chokes have been considered since those cores exhibit the best performance to size/weight ratio [16].

\section{Filter Optimization}

The design of the individual filter topologies has been optimized in order to obtain a good trade-off between weight and losses where the weight is considered as the more critical design issue. The core size and the wire selection have been free variables to obtain the inductance value obtained with 3, i.e. $(\mathrm{P}=$ power losses, $\mathrm{W}=$ weight $)$

$$
\min _{\substack{\text { core-size } \\ \text { wire-size }}}\left(k_{W} W_{\text {tot }}+k_{P} P_{\text {tot }}\right)
$$

with

$$
W_{\text {tot }}=W_{\text {cores }}+W_{\text {wire }}+W_{\text {capacitors }}
$$

and

$$
P_{\text {tot }}=P_{\text {cores }}+P_{\text {wire }}+P_{\text {capacitors }}
$$

The calculated weights and losses of different filter structures with varying number of filter stages ("N+M" = 1..4) (with "N+M" defined as before, i.e. $\mathrm{N}$ the number of common filter stages and $M$ the number of individual filter stages) and the different distribution schemes are shown in Figs. 11 and 12. On can observe that a single filter stage leads in both cases ("1+0" and "0+1") to a very bulky and heavy design and considerable improvements can be achieved using a higher number of filter stages. Independently of the distribution scheme, the optimal number of filter stages w.r.t. weight is always three ("3+0", " $0+3 ", " 1+2 ")$ with a total weight close to $2.5 \mathrm{~kg}$. The existence of such an optimum in the number

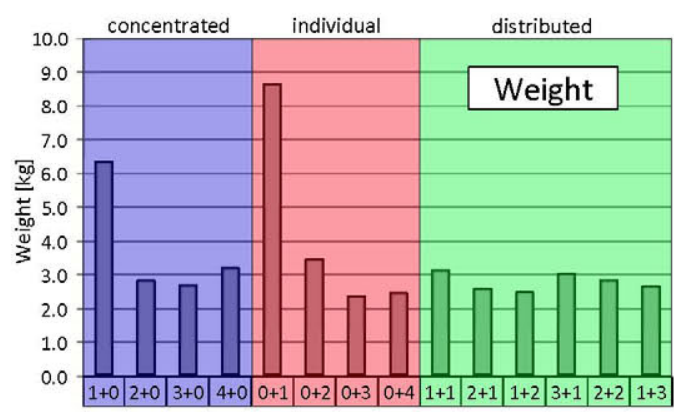

Fig. 11. Optimal weight of different input filter configurations - different number of stages and different distribution schemes

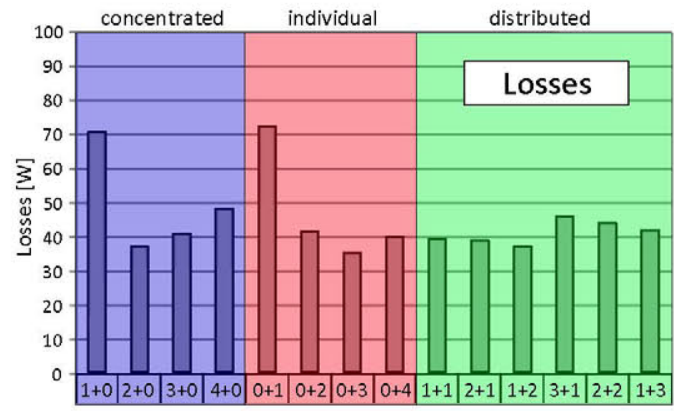

Fig. 12. Losses of the different input filter configurations - different number of stages and different distribution schemes
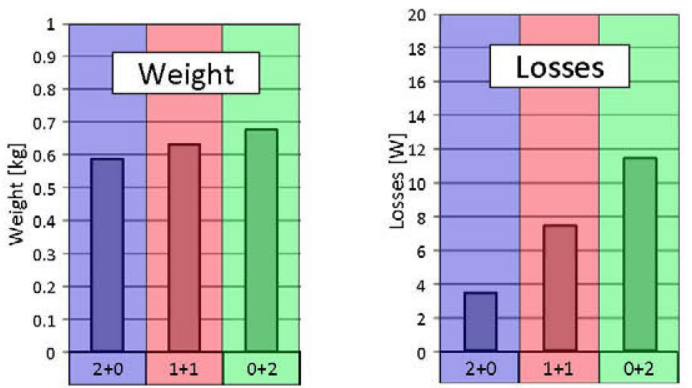

Fig. 13. Weight and losses of different distribution schemes of a two-stage $\mathrm{CM}$ filter employing commercially available components [16]

of filter stages can be explained in each case by the tradeoff between the smaller components which can be employed in multi-stage filters and the increasing number of components. Moreover the analysis shows that in all cases the optima are very close to each other. This shows that even though in case that individual filter stages are employed using components with lower current rating (e.g. smaller cores and thinner wire), the improvements in weight are compensated by the increased number of filter components. 
TABLE II

INPUT FILTER COMPONENTS OF COMMON FILTER STAGE

\begin{tabular}{|l|c|}
\hline DM Inductor & Magnetics 58439 High Flux Powder Core [15] \\
\hline CM inductor & VAC T60405-S6123-X363 [16] \\
\hline DM Capacitor & EPCOS $2.2 \mu$ F Film Capacitor \\
\hline CM capacitor & Murata $2 \times 2.2$ nF Y-capacitor \\
\hline
\end{tabular}

TABLE III

INPUT FILTER COMPONENTS OF INDIVIDUAL FILTER STAGE

\begin{tabular}{|l|c|}
\hline DM Inductor & Magnetics 58548 High Flux Powder Core [15] \\
\hline CM inductor & VAC T60405-S6123-X317 [16] \\
\hline DM Capacitor & EPCOS $1.0 \mu$ F Film Capacitor \\
\hline CM capacitor & Murata $2.2 \mathrm{nF}$ Y-capacitor \\
\hline
\end{tabular}

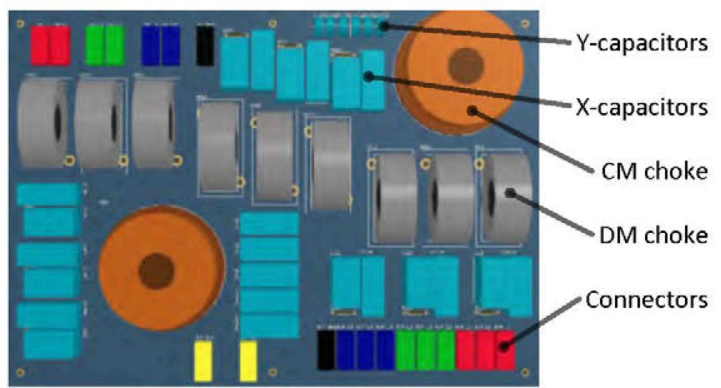

Fig. 14. CAD drawing of a three-stage DM / two-stage CM input filter for all three converter modules

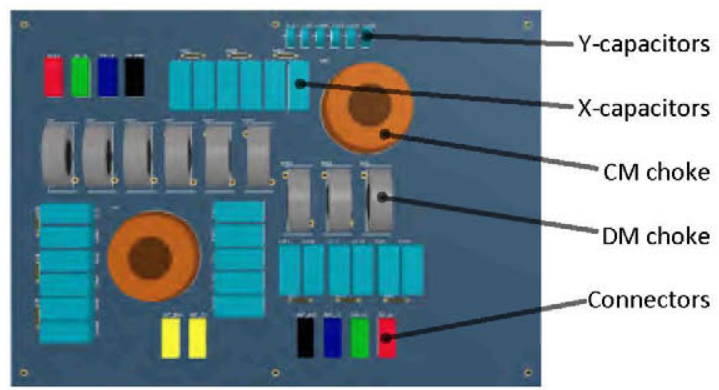

Fig. 15. CAD-drawing of a three-stage DM / two-stage CM input filter for a single converter module

\section{Filter Implementation and Verification}

For experimental validation of the results, different input filters have been designed. Fig.14 shows a drawing of a completely concentrated filter solution with three DM filter stages and $2 \mathrm{CM}$ filter stages(DM " $3+0$ " and CM " $2+0 "$ "). Fig.15 shows an input filter for one individual converter cell, also consisting of 3 DM stages and 2 CM stages (DM " $0+3$ " and CM " $0+2$ "), note that the complete input filter would consist of three of these modules. The employed components are shown in Tables II and III

\section{CONCLUSION}

The importance of a correct model of the line impedance stabilization network and the accurate way to measure the input current spectrum has been addressed. This issue can be and often is neglected in conducted EMI simulations for residential/industrial equipment where the frequency range of interest starts at $150 \mathrm{kHz}$. For aerospace applications however, where the frequency range of interest starts at $10 \mathrm{kHz}$, it needs to be considered, otherwise the noise spectrum will be estimated too high leading to a suboptimal input filter design.

Based on simulated noise spectra of a modular three-phase PWM rectifier system, an input filter has been designed. In these simulations the effect of a possible power unbalance between the individual converter modules has been considered. The possible degrees of distribution of the input filter between fully concentrated filter solution and individual filter solutions for the converter modules has been discussed and an optimal design w.r.t. weight has been proposed, considering the degree of distribution, the number of filter stages and the tradeoff between component weight and losses. The optimal number of filter stages has been determined to be three, independent of the degree of distribution.

\section{REFERENCES}

[1] T. Carter, "Switch mode power supplies: and EMI engineer's point of view", Proceedings of Southcon, 1994

[2] L. Tihanyi, "Electromagnetic compatibility in power electronics", IEEE Press / butterworth-Heinemann, 1995

[3] H. W. Ott, "Electromagnetic compatibility engineering", John Wiley \& Sons, 2009

[4] C.I.S.P.R., "Industrial, scientific and medical (ISM) radio-frequency equipment - electromagnetic disturbance characteristics - limits and methods of measurement", Publication 11, Geneva, Switzerland, IEC International Special Committee on Radio Interference

[5] C.I.S.P.R., "Information technology equipment - radio disturvbance characteristics - limits and methods of measurement", Publication 22 Geneva, Switzerland, IEC International Standard Committee on Radio Interference

[6] MIL-STD-461E, "Department of defense interface standard requirements for the control of electromagnetic interference characteristics of subsystems and equipment", August 1999

[7] K. Mainali, R.Oruganti, "Conducted EMI mitigation techniques for switch-mode power converters: a survey", IEEE Transactions on Power Electronics, Vol. 25, No. 9, September 2009, pp. 2344-2356

[8] M. L. Heldwein, J. W. Kolar, "Impact of EMC filters on the power desnsity of modern three-phase PWM converters", IEEE Transactions on Power Electronics, Vol. 24, No. 6, June 2006, pp. 1577-1588

[9] A. Stupar, T. Fridli,J. Miniböck, M. Schweizer, J. W. Kolar, "Towards a 99\% efficient three-phase buck-type PFC rectifier for a $400 \mathrm{~V} \mathrm{DC}$ distribution system", Proceedings of the 26th Annual Applied Power Eclectronics Conference and Exposition (APEC), 2011, pp. 505-512

[10] T. Nussbaumer, M. L. Heldwein, J. W. Kolar, "Differential mode input filter design for a three-phase buck-type PWm rectifier based on modeling of the EMC test receiver", IEEE Transactions on Industrial Electronics, Vol. 53, No. 5, October 2006, pp. 1649-1661

[11] M. L. Heldwein, J. W. Kolar, "Design of minimum volume EMC input filters for an ultra compact three-phase PWM rectifier", Proceedings of the 9th Brazilian Power Electronics Conference (COBEP), 2007

[12] M. Silva, N. Hensgens, J. Oliver, P. Alou, O. García, J. A. Cobos, New considerations in the input filter design of a three-phase buck-type PWM rectifier for aircraft applications, Proceedings of the 3rd Annual IEEE Energy Conversion Congress and Exposition (ECCE), 2011, pp. 40874092

[13] R. W. Erickson, "Optimal single resistor damping of input filters", Proceedings of the 14th Annual IEEE Applied Power Electronics Conference and Exposition (APEC), 1999, pp. 1073-1079

[14] L. Xing, J. Sun, "Optimal damping of multi-stage EMI filters", Proceedings of the 26th Annual IEEE Applied Power Electronics Conference and Exposition (APEC), 2011, pp. 1721-1728

[15] Magnetics Powder Cores catalog 2011, available online: www.maginc.com

[16] Vacuumschmelze Nanocrystalline Vitroperm EMC products Catalog, available online: www.vacuumschmelze.de 